\title{
K-mer-based Machine Learning method to classify LTR- retrotransposons in plant genomes
}

\author{
Simon Orozco-Arias ${ }^{\text {Corresp., } 1,2}{ }^{2}$, Mariana Candamil ${ }^{1}$, Paula Jaimes ${ }^{1}$, Johan Pina ${ }^{1}$, Reinel Tabares-Soto ${ }^{3}$, Romain Guyot \\ 3, 4 , Gustavo Isaza ${ }^{\text {Corresp. } 2}$ \\ ${ }^{1}$ Department of Computer Science, Universidad Autónoma de Manizales, Manizales, Caldas, Colombia \\ Department of Systems and Informatics, Universidad de Caldas, Manizales, Caldas, Colombia \\ 3 Department of Electronics and Automation, Universidad Autónoma de Manizales, Manizales, Caldas, Colombia \\ 4 Institut de Recherche pour le Développement, CIRAD, Univ. Montpellier, Montpellier, France \\ Corresponding Authors: Simon Orozco-Arias, Gustavo Isaza \\ Email address: simon.orozco.arias@gmail.com, gustavo.isaza@ucaldas.edu.co
}

Every day more plant genomes are available in public databases and additional massive sequencing projects (i.e., that aim to sequence thousands of individuals) are formulated and released. Nevertheless, there are not enough automatic tools to analyze this large amount of genomic information. LTR retrotransposons are the most frequent repetitive sequences in plant genomes; however, their detection and classification are commonly performed using semi-automatic and time-consuming programs. Despite the availability of several bioinformatic tools that follow different approaches to detect and classify them, none of these tools can individually obtain accurate results. Here, we used Machine Learning algorithms based on $k$-mer counts to classify LTR retrotransposons from other genomic sequences and into lineages/families with an F1-Score of 95\%, contributing to develop a free-alignment and automatic method to analyze these sequences. 


\title{
1 K-mer-based Machine Learning method to classify
}

2

3

4

5

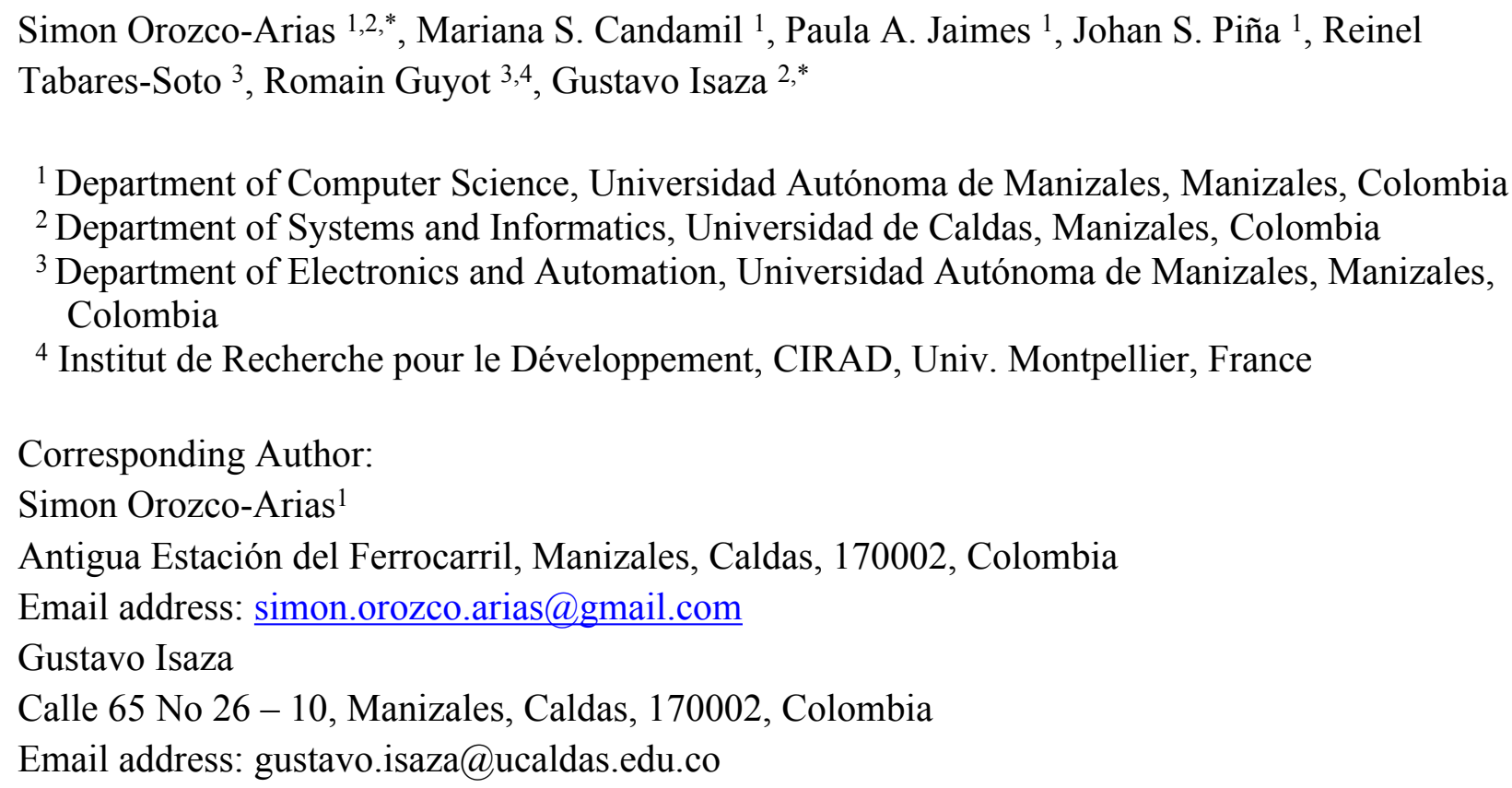

\begin{abstract}
Every day more plant genomes are available in public databases and additional massive sequencing projects (i.e., that aim to sequence thousands of individuals) are formulated and released. Nevertheless, there are not enough automatic tools to analyze this large amount of genomic information. LTR retrotransposons are the most frequent repetitive sequences in plant genomes; however, their detection and classification are commonly performed using semiautomatic and time-consuming programs. Despite the availability of several bioinformatic tools that follow different approaches to detect and classify them, none of these tools can individually obtain accurate results. Here, we used Machine Learning algorithms based on $k$-mer counts to classify LTR retrotransposons from other genomic sequences and into lineages/families with an F1-Score of $95 \%$, contributing to develop a free-alignment and automatic method to analyze these sequences.
\end{abstract}

Keywords: transposable elements, LTR retrotransposons, plant genomes, machine learning, classification, free-alignment approach, $k$-mer based method.

\section{Introduction}

The availability of large-scale biological data is changing the way researchers must analyze and find solutions to problems in almost every area of biological sciences. Machine Learning (ML) algorithms can use this data to automatically learn the parameters needed to fit a model to a 
42 specific problem (Shastry \& Sanjay, 2020) in order to predict known labels. This process is

43 called supervised learning (Zou et al., 2018). Bioinformatics, which is an intersection between

44 computer sciences, biological sciences, and mathematics (Orozco-Arias et al., 2017), plays a

45 central role in storing, analyzing, categorizing, and labeling the huge flow of information

46 generated, for example, by next-generation sequencing (NGS) platforms. Advances in these

47 sequencing technologies have provided a new paradigm in the field of post-genomics (Rigal \&

48 Mathieu, 2011; Chen et al., 2014; Rishishwar et al., 2017), which focuses on how to store,

49 process, and analyze these data streams in acceptable time frames (Rigal \& Mathieu, 2011; Chen

50 et al., 2014; Rishishwar et al., 2017).

51 By automatizing tasks that were done manually, ML is impacting many areas of bioinformatics

52 (Li et al., 2020), such as genomics (Libbrecht \& Noble, 2015; Zou et al., 2018; Eraslan et al.,

53 2019), systems biology (Larrañaga et al., 2006), and, specifically, the annotation of transposable

54 elements (TEs) (Orozco-Arias et al., 2019). This last task is a current challenge in genomics (Ou,

55 Chen \& Jiang, 2018; Orozco-Arias et al., 2020). There is a growing interest in these repeated

56 sequences due to their key functional and evolutionary roles on eukaryote genomes (Hesam \&

57 Ali, 2010; Orozco-Arias, Isaza \& Guyot, 2019). Nevertheless, the detection and classification of

58 these sequences remain complex because of their highly repetitive nature, diversity,

59 polymorphism, species specificity, among other factors (Ou, Chen \& Jiang, 2018; Mustafin \&

60 Khusnutdinova, 2018).

61 Although there is an open debate on how to classify TEs, the most classification approach is

62 based on their lifecycle (i.e., how they move inside the genome). TEs are classified hierarchically

63 (Orozco-Arias et al., 2019); first, they are divided into two main classes (e.g., Class I or

64 retrotransposons and Class II or DNA transposons (Wicker et al., 2007)) and further divided into

65 orders according to the similarity of their coding domains (Chaparro et al., 2015; Orozco-Arias,

66 Isaza \& Guyot, 2019; Neumann et al., 2019). In plants, the most frequent elements are, by far,

67 long terminal repeat (LTR) retrotransposons (LTR-RTs, an order from Class I) (Gao et al., 2012;

68 Grandbastien, 2015a), which account for $80 \%$ of the genome size of species such as wheat,

69 barley, or the rubber tree (Rahman et al., 2013).

70 Indeed, bioinformatic approaches have developed many tools to detect and classify transposable

71 elements, including multiple approaches that group TEs based mainly on their structure,

72 homology, redundancy, or conservation across genomes (Rawal \& Ramaswamy, 2011; Jiang \&

73 Ramachandran, 2013; Loureiro et al., 2013a; Schietgat et al., 2018a). Nevertheless, the

74 complexity of these elements does not consistently allow for accurate, reliable, and reproducible

75 results across programs for all types of TEs (Arkhipova, 2017). In recent years, several machine

76 learning-based methods have been proposed and evaluated, which take advantage of the

77 thousands of sequences available in several datasets, such as Repbase (Jurka et al., 2005),

78 RepetDB (Cornut et al., 2019), PGSB (Spannagl et al., 2016a), and InpactorDB (Orozco-Arias et

79 al., 2021).

80 Several studies have, therefore, proposed the use of ML for TE analysis (reviewed in (Orozco-

81 Arias et al., 2019)) to differentiate between LTR-RTs and SINEs (Ashlock \& Datta, 2012) or 
82 autonomous and non-autonomous LTR-RTs in the Coffea canephora genome (Arango-López et

83 al., 2017), and to improve the accuracy and performance of the classification (Loureiro et al.,

84 2013a; Nakano et al., 2017). Recently, a random forest algorithm was used to broadly classify

85 LTR-RTs into superfamilies (Schietgat et al., 2018a), while additional pre-processing techniques

86 and coding schemes allow their deep classification (Orozco-Arias et al., 2020). Moreover,

87 several deep neural network (DNN) architectures that perform TE classification have been

88 published so far. For instance, Nakano et al. implemented a fully connected neural network

89 (FNN) (Nakano et al., 2018a), da Cruz et al. used a convolutional neural network (CNN) with a

90 2D representation of the sequences (da Cruz et al., 2019), and Yan et al. used a CNN in 1D to

91 classify TEs into superfamilies (da Cruz et al., 2020; Yan, Bombarely \& Li, 2020).

92

93

Despite these efforts, none consider the need to both detect and classify elements at the same time, or specifically aim to classify LTR-RTs to the lineage/family level. In this paper, we evaluate and demonstrate the application of ML algorithms in the binary classification between

LTR-RTs and other genomic features, multi-class classification into lineages/families or both (in a single process), as well as the importance of the features used, in order to design a freealignment method for the annotation of LTR-RT in plant genomes based on $k$-mer frequencies.

\section{Materials \& Methods}

\section{Dataset composition}

102

We used InpactorDB ((Orozco-Arias et al., 2021), doi: 10.5281/zenodo.4386316 or 10.23708/QCMOUA), which comprises 67,241 LTR retrotransposon sequences, deeply classified into lineages/families, from 195 plant species. This dataset initially contained sequences from Repbase, RepetDB, and PGSB, which were processed using several filters to remove low quality elements (i.e., elements with nested sequences) (Orozco-Arias et al., 2021). It also contained LTR-RTs predicted by LTR_STRUC (McCarthy \& McDonald, 2003) and EDTA (Ou et al., 2019). As negative instances, we created a dataset composed of annotated genomic features other than LTR_RTs, such as coding sequences (CDS), different types of RNA (e.g., mRNA, tRNA, non-coding RNA, among others), and other types of transposable elements that do not belong to LTR-RTs (e.g. TIR, Helitron, PLEs, DIRs, LINEs, and SINEs) from the same plant species contained in InpactorDB. These additional TE sequences were available in databases such as PGSB PlantsDB (Spannagl et al., 2016b), Repbase (v. 20.05, 2017) (Bao, Kojima \& Kohany, 2015), RepetDB (Amselem et al., 2019), Ensembl Plants (Bolser et al., 2017), and JGI (Joint Genome Institute) (Nordberg et al., 2014) (Supplementary Material 1)).

116 For the binary classification task, we randomly selected 10,000 LTR retrotransposon sequences 117 (taken as positive instances) and 10,000 genomic feature sequences for the negative instances. 118 For the classification task into lineages/families, we used only InpactorDB data, while, for the 119 binary plus multi-class classification problem (unified at a single ML process), we filtered the negative instances to retain only sequences longer than $6 \mathrm{~Kb}$. We did this filter in order to reduce the number of sequences from more than 3 million to 34,830 and because the average length of 
122 Copia elements in InpactorDB is 5,957.48. In contrast the average length of Gypsy elements is $12310,760.57$.

124 As features, we selected $k$-mer frequencies with $1<=\mathrm{k}<=6$, as recommended in (Orozco-Arias et 125 al., 2020), calculating all possible $k$-mers, and later counting the number of occurrences of them 126 in each sequence. We calculated them for lineage-level classification and the binary plus multi127 class classification task. For binary classification, we used the same coding schemes as 128 implemented in (Orozco-Arias et al., 2020), such as DAX (Yu et al., 2015), EIIP (Nair \& 129 Sreenadhan, 2006), Complementary (Akhtar, Epps \& Ambikairajah, 2008), Enthalpy (Kauer \& 130 Blöcker, 2003), and Galois (4) (Rosen, 2006). Additionally, two techniques were applied to 131 automatically extract features from the sequences: i) $k$-mer frequencies were obtained for each element and ii) three physical-chemical (PC) properties were extracted, such as average hydrogen bonding energy per base pair (bp), stacking energy (per bp), and solvation energy (per bp), which were calculated by taking the first di-nucleotide and moving in a sliding window of one base at a time (Jaiswal \& Krishnamachari, 2019). Moreover, we pre-processed the data by scaling, following the strategy implemented in (Tabares-soto et al., 2020), and performed a dimensional reduction through a principal component analysis (PCA) (Wold, Esbensen \& Geladi, 1987) with a cumulative variance of $96 \%$ and tolerance of $1 \mathrm{e}-4$. For the binary classification task, we divided the dataset into a training set ( $80 \%$ of the data) a validation set (10\%), and a test set (10\%). For multi-class classification into lineages, we used the same partition and additionally, we used k-cross-validation (Komer, Bergstra \& Eliasmith, 2014) with $\mathrm{k}=9$ after tuning hyper-parameters in order to test the generalization property of each model.

144

\section{Machine Learning algorithms used}

For binary classification between positive (LTR retrotransposons) and negative (other genomic features) instances, we used the same algorithms described in (Orozco-Arias et al., 2020); thus, we used Linear Support Vector Classifier (SVC), Logistic Regression (LR), Linear Discriminant Analysis (LDA), K-Nearest Neighbors (KNN), Naive Bayesian Classifier (NB), Multi-Layer Perceptron (MLP), Decision Trees (DT), and Random Forest (RF) and selected the larger F1Score for different values of a hyper-parameter (as in (Orozco-Arias et al., 2020)). For classification, we used the supervised models that showed the best performance in (OrozcoArias et al., 2020), such as KNN, LR, SVC, and LDA, but we applied hyper-parameter tuning (Table 1) using GridSearchCV from Scikit-learn (Pedregosa et al., 2011), using only sequences from InpactorDB (we did not include negative instances due to the high memory required). We used the F1-Score as a performance metric in all executions since it is not affected much by unbalanced datasets such as LTR-RTs (Orozco-Arias et al., 2020). Next, a Stacking Classifier was implemented as an ensemble algorithm, which is a combination of multiple ML models for creating a more complex model (Zhang \& Ma, 2012; Müller, Guido \& others, 2016). The stacking classifier comprised LDA, Linear SVC, and KNN algorithms and used Random Forest as a meta-classifier. Similarly, for binary plus multi-class classification, we 
162

163

164

165

166

167

168

169

170

171

172

173

174

175

176

177

178

179

180

181

182

183

184

185

186

187

188

189

190

191

192

193

194

195

196

197

198

199

200

201

used a Stacking Classifier with KNN, LDA, and LR algorithms and Random Forest as a metaclassifier. Figure 1 summarizes the three approaches used in this study. The implementation in Python 3 of all algorithms used in this study is available in the Supplementary Material 2 or at https://github.com/simonorozcoarias/MachineLearningInTEs/blob/master/Scripts/binary_plus_m ulti_clasification.py

\section{Feature selection}

We used the Gradient Boosting algorithm (Friedman, 2002) implemented in Scikit-Learn to determine the importance of each feature using the complete dataset (InpactorDB plus negative instances). Gradient Boosting generates scores for each feature that represent how useful it was in the construction of the boosted decision trees. The more valuable the feature is for making key decisions, the higher its importance score (Hastie, Tibshirani \& Friedman, 2009). Thus, we extracted features with an importance score greater than 60, 40, 30, 20, 10, which yielded 65, 289, 508, 1034, and 2397 features from 5,460 $k$-mers. We used the following hyper-parameters: boosting_type $=$ 'goss', n_estimators $=10000$, and class_weight='balance'. Finally, we extracted the selected features in order to create new reduced datasets that were used to train the same ensemble algorithm implemented in the binary plus multi-class classification task.

\section{Results}

\section{Binary classification of LTR retrotransposons and other genomic features}

As negative instances, we obtained 2,713,028 coding sequences (CDS), 262,925 RNAs of different types (e.g., ncRNA, mRNA, miRNA, rRNA, snRNA, and tRNA), 37,077 TEs that did not correspond to LTR retrotransposons (i.e. TEs class II, LINEs, SINEs, DIRS, and PLEs), and 3566 quimeric sequences from Repbase, PGSB, and RepetDB (Table 2). These sequences, with the exception of TEs (outside LTR-RTs), were obtained from 47 plant species available in public databases, such as Ensembl Plants and JGI (Supplementary Material 1). We used sequences from InpactorDB as positive instances. Due to the high imbalance between the two instances (3,016,596 negative vs 67,241 positive), we randomly extracted 10,000 sequences from each class. Then, the DNA sequences were converted to numerical representations using the coding schemes and automated techniques described in (Orozco-Arias et al., 2020). Finally, we applied data scaling and a dimensional reduction through PCA (Tabares-soto et al., 2020). Using this dataset, we trained ML algorithms and determined their performance in terms of the F1-Score of each coding scheme over each ML model (Figure 2). For the binary classification task, we obtained F1-Scores up to $97.9 \% 96.3 \%$, and $95.9 \%$ for MLP, SVC, and LR, respectively, in the test dataset using $k$-mer frequencies as features.

\section{Multi-class classification of LTR retrotransposons into lineages/families}

For each of the selected models, a dictionary was created containing the hyper-parameters and the values to be iterated. After training each ML algorithm with GridsearchCV (Tabares-soto et al., 2020), we determined the parameters that generated the best performance, as shown in Table 
202

203

204

205

206

207

208

209

210

211

212

213

214

215

216

217

218

219

220

221

222

223

224

225

226

227

228

229

230

231

232

233

234

235

236

237

238

239

240

241

3. After tuning the hyper-parameters, each model was retrained to determine its performance. We obtained F1-Scores of $91 \%, 97 \%, 96 \%$, and 97\% with LR, KNN, LDA, and SVC algorithms, respectively (Figure 3 ).

For the ensemble algorithm, the LR classifier was excluded since it showed the lowest performance (Figure 3A). Therefore, the Stacking Classifier was implemented as an ensemble algorithm, composed of LDA, Linear SVC, and KNN algorithms, using Random Forest as metaclassifier. The performance of this ensemble model resulted in a 97\% F1-Score, accuracy, recall, precision (Figure 4), and 99\% in area under ROC (receiver operating characteristic) curve (AUC) (Figure S1) for the classification of LTR retrotransposons.

\section{Binary plus multi-class classification task}

After obtaining promising results in both the binary and multi-class classification tasks, we proceeded to merge them into a single ML problem. Thus, we included the negative instances as another class but deleted the sequences with a length of less than $6 \mathrm{~Kb}$ (Table 4). Furthermore, only $k$-mers frequencies were used as features because of the high performance obtained for the two problems separately. We also used the hyper-parameter values tuned (Table 3) for KNN, LDA, and LR (Figure 5). Finally, F1-Scores of 95\%, 94\%, and 84\% were obtained using KNN, LDA, and LR, respectively.

We implemented an ensemble method (the same implemented for the classification task) using the three algorithms aforementioned and used RF as a meta-classifier. We obtained an F1-Score of $96 \%$ in $\mathrm{k}$-cross validation with $\mathrm{k}=9$ (Figure 6). This method also obtained $95 \%$ of precision and recall and 98\% in AUC (Figure S2). Furthermore, as shown in Figure 7, the classes with the lowest F1-Scores are Class 8 (Ikeros, Copia) and 16 (Galadriel, Gypsy) since these classes have the lowest number of samples.

\section{Feature selection and evaluation}

Using the Gradient Boosting algorithm and the entire dataset (negative instances plus InpactorDB), we obtained the importance of each feature ( $k$-mers frequencies). The number of features is relatively high $(5,460)$. Since the computational cost to process them can be very high, the number of features must be reduced without reducing the performance of the ML algorithm. Figure 8 shows the importance of all features determined by Gradient Boosting. The results displayed in Figure 8 demonstrate that some features are not relevant to the binary plus multi-class classification task. We extracted those with an importance score greater or equal to 40, thus, retaining only 289 features out of 5,460 (5.29\%). The 10 most important features are: A, T, AAAAAA, ATAT, AGGGGG, CCCCC, TTTTTT, AGCT, GATC, GATGA with importance scores of 199, 179.5, 165, 140, 132.5, 132, 125.5, 124, 124, 114.5, respectively. Among the 289 selected features, we observed that increasing the length of $\mathrm{K}$ decreases the percentage of top selected features with greater importance (Figure 9). In total, the 289 selected features were composed of 4, 10, 32, 109, 97, 37 of $k$-mers generated using $\mathrm{k}=1,2,3,4,5$ and 6 , respectively (Supplementary Material 3). 
242 Then, we executed the ensemble method again using the reduced dataset containing the most 243 important features. We also test different importance score thresholds in order to keep different 244 number of features. We used the same pre-processing technique and hyper-parameter values of 245 the previous execution. The results show that reducing the number of features to $1.73 \%$ (from 2465,460 to 95 ), 5.29\% (289 features), 9.3\% (508 features), 18.93\% (1034 features), and 43.9\% 247 (2397 features), did not considerably decrease performance, as indicated by a 93.5\%, 95.2\%, $24895.6 \%, 95.4 \%, 95.6 \% \mathrm{~F} 1$-Score (Figure 10), accuracy, recall, and precision, using as importance 249 score threshold $60,40,30,20$, and 10 respectively. We also noted that using 289 features we 250 obtained an $97 \%$ in AUC (Figure S3).

251

\section{Discussion}

253

254

255

256

257

258

259

260

261

262

263

264

265

266

267

268

269

270

271

272

273

274

275

276

277

278

279

280

281

Transposable element detection and classification are time-consuming tasks for plant genome annotation projects and involve semi-automatic pipelines and curation by experts. These pipelines generally rely on sequence alignment approaches, which have a significant computational cost in the analysis of large genomes or TE compound genomes. The development of automatic algorithms for TE detection and annotation, without sequence alignment, is now required to process the huge amount of genomic information that is being generated.

The most frequent repeated sequences in plant genomes are LTR retrotransposons (Gao et al., 2012; Grandbastien, 2015b) Their transcriptional activities and mobilities can have a profound impact on the structure, composition, and size of genomes, among others (Orozco-Arias, Isaza \& Guyot, 2019). To detect these elements in assembled genome sequences, the most frequent methods are based on their specific internal structure (e.g., the duplication of their LTR at both ends of the element) and domains, or via homology searches against reference libraries (Loureiro et al., 2013b; Nicolas, Peterlongo \& Tempel, 2016). Nevertheless, these strategies have strong limitations (Orozco-Arias, Isaza \& Guyot, 2019). Structure-based methods will not accurately detect partial elements, variations, or non-autonomous elements such as solo-LTR, TR-GAG (Chaparro et al., 2015), or TRIM (Witte et al., 2001). Moreover, homology-based methods can induce low quality annotations if the reference library is incomplete or constructed with distant species of the species to be annotated (Orozco-Arias, Isaza \& Guyot, 2019). These problems can be overcome using new methods, such as machine learning methods, that are not based on structure or homology (i.e., sequence alignment) (Nakano et al., 2018b; Schietgat et al., 2018b; Orozco-Arias et al., 2019; da Cruz et al., 2020). Although some studies have used ML to analyzed TEs, none of them have focused on the detection and lineage/family level classification of LTR retrotransposons. In previous studies (Orozco-Arias et al., 2020, 2021), we have shown that the classification of these elements can be very efficient with ML approaches but that coding schemes and feature extraction techniques can deeply influence the performance of such algorithms.

Here, we have shown the possibility of both binary and multi-class classification through ML with different approaches. First, by using a negative dataset (i.e., sequences of genomic features excluding LTR-RTs), we obtained an F1-Score up to $97.9 \%$ in the binary classification task. In

Peer) reviewing PDF | (2021:02:58021:1:0:NEW 12 Apr 2021) 
282 the detailed lineage/family level classification task, we obtained a performance of $97 \%$ by using

283 the same datasets as in (Orozco-Arias et al., 2020) but including more elements from

284 InpactorDB. Finally, we unified both tasks into a single process, obtaining a 96\% F1-Score.

285 These results were achieved using the $k$-mer frequencies of sequences. $K$-mers are frequently

286 used in bioinformatics in the areas of quality control of generated sequences (Mapleson et al.,

287 2017), metagenomics (Breitwieser, Baker \& Salzberg, 2018), de novo assembly of genomes and 288 transcriptomes (Zerbino \& Birney, 2008; Simpson et al., 2009), genome size estimation (Sun et 289 al., 2018), and de novo detection of transposable elements (Price, Jones \& Pevzner, 2005). In 290 addition to detection, $k$-mers have also demonstrated their usefulness in higher hierarchical 291 classification approaches (at the superfamily level). Nakano and colleagues (Nakano et al., 292 2018a) exploited the characteristics of $k$-mer frequencies (with $\mathrm{k}=2,3,4$ ) as features to train a 293 deep neural network, and Yan and colleagues [38] demonstrated interesting statistical differences 294 using $k$-mer frequencies with $\mathrm{k}$ ranging from 3 to 7 . Here, we found that the combination of 295 certain $k$-mer frequencies with different values of $\mathrm{k}$ (from 1 to 6 ) added reliability to the binary 296 plus multi-class classification task, requiring only 289 features (out of the initial 5,460 features) 297 to obtain an F1-Score of 95\%. We also demonstrated that, among all possible $k$-mers generated, 298 larger $\mathrm{k}$ values reduce the number of features with high importance scores. The specific $k$-mer 299 that contributes the most to the binary and multi-class classification characteristics must be 300 analyzed in detail to understand its involvement in the composition and structure of the elements 301 of each lineage. This work highlights the feasibility of designing and implementing ML-based 302 tools that automate the complex process of annotating LTR retrotransposons in plant genomes. 303 As future work, we propose the implementation of benchmarking to compare this method with 304 well-established methods such as those based on homology and structure in various plant 305 genomes.

306

\section{Conclusions}

308 Massive sequencing projects require automatic tools to analyze large amounts of genomic 309 information in a fast yet accurate, reliable, and reproducible manner. The binary classification of 310 LTR-RTs and other genomic features and lineage-level classification of them in plant genomes 311 can be performed using ML-based and ensemble methods, demonstrating good performance (up 312 to $96 \% \mathrm{~F} 1-\mathrm{Score}$ ). This task can be performed with only $289 k$-mer frequencies, allowing low 313 computational resources and time. These results can be used in the design and implementation of 314 automatic and alignment-free tools to solve the issue of processing the increasing number of 315 available plant genomes.

316

\section{Funding}

318 Simon Orozco-Arias is supported by a Ph.D. grant from the Ministry of Science, Technology and 319 Innovation (Minciencias) of Colombia, Grant Call 785/2017. The authors and publication fees 320 were supported by Universidad Autónoma de Manizales, Manizales, Colombia under project 321 589-089. This work was supported by Ecos-Nord NC21MA01 and STICAMSUC 21-STIC-13. 
323

324

325

326

327

328

329

330

331

332

333

334

335

336

337

338

339

340

341

342

343

344

345

346

347

348

349

350

351

352

353

354

355

356

357

358

359

360

361

362

363

364

365

366

367

\section{References}

Akhtar M, Epps J, Ambikairajah E. 2008. Signal processing in sequence analysis: advances in eukaryotic gene prediction. IEEE journal of selected topics in signal processing 2:310-321.

Amselem J, Cornut G, Choisne N, Alaux M, Alfama-Depauw F, Jamilloux V, Maumus F, Letellier T, Luyten I, Pommier C, Adam-Blondon AF, Quesneville H. 2019. RepetDB: A unified resource for transposable element references. Mobile DNA 10:4-11. DOI: 10.1186/s13100-019-0150-y.

Arango-López J, Orozco-Arias S, Salazar JA, Guyot R, Arango-Lopez J, Orozco-Arias S, Salazar JA, Guyot R. 2017. Application of Data Mining Algorithms to Classify Biological Data: The Coffea canephora Genome Case. In: Communications in Computer and Information Science. Cali, Colombia, 156-170. DOI: 10.1007/978-3-319-66562-7_12.

Arkhipova IR. 2017. Using bioinformatic and phylogenetic approaches to classify transposable elements and understand their complex evolutionary histories. Mobile DNA 8:19. DOI: 10.1186/s13100-017-0103-2.

Ashlock W, Datta S. 2012. Distinguishing endogenous retroviral LTRs from SINE elements using features extracted from evolved side effect machines. IEEE/ACM Transactions on Computational Biology and Bioinformatics 9:1676-1689. DOI: 10.1109/TCBB.2012.116.

Bao W, Kojima KK, Kohany O. 2015. Repbase Update, a database of repetitive elements in eukaryotic genomes. Mobile DNA 6:4-9. DOI: 10.1186/s13100-015-0041-9.

Bolser DM, Staines DM, Perry E, Kersey PJ. 2017. Ensembl plants: Integrating tools for visualizing, mining, and analyzing plant genomic data. Methods in Molecular Biology 1533:1-31. DOI: 10.1007/978-1-4939-6658-5_1.

Breitwieser FP, Baker DN, Salzberg SL. 2018. KrakenUniq: confident and fast metagenomics classification using unique k-mer counts. Genome biology 19:1-10.

Chaparro C, Gayraud T, De Souza RF, Domingues DS, Akaffou SS, Vanzela ALL, De Kochko A, Rigoreau M, Crouzillat D, Hamon S, Hamon P, Guyot R, Laforga Vanzela AL, Kochko A de, Rigoreau M, Crouzillat D, Hamon S, Hamon P, Guyot R. 2015. Terminal-repeat retrotransposons with GAG domain in plant genomes: A new testimony on the complex world of transposable elements. Genome Biology and Evolution 7:493-504. DOI: 10.1093/gbe/evv001.

Chen W, Feng PM, Deng EZ, Lin H, Chou KC. 2014. iTIS-PseTNC: A sequence-based predictor for identifying translation initiation site in human genes using pseudo trinucleotide composition. Analytical Biochemistry 462:76-83. DOI: 10.1016/j.ab.2014.06.022.

Cornut G, Choisne N, Alaux M, Alfama-Depauw F, Jamilloux V, Maumus F, Letellier T, Luyten I, Pommier C, Adam-Blondon A-F, others. 2019. RepetDB: a unified resource for transposable element references. Mobile DNA 10.

da Cruz MHP, Domingues DS, Saito PTM, Paschoal AR, Bugatti PH. 2020. TERL: Classification of Transposable Elements by Convolutional Neural Networks. Briefings in Bioinformatics:19. DOI: https://doi.org/10.1093/bib/bbaa185.

da Cruz MHP, Saito PTM, Paschoal AR, Bugatti PH. 2019. Classification of Transposable Elements by Convolutional Neural Networks. In: Lecture Notes in Computer Science. Springer International Publishing, 157-168. DOI: 10.1201/9781420049138.

Eraslan G, Avsec Ž, Gagneur J, Theis FJ. 2019. Deep learning: new computational modelling techniques for genomics. Nature Reviews Genetics. DOI: 10.1038/s41576-019-0122-6.

Friedman JH. 2002. Stochastic gradient boosting. Computational statistics \& data analysis

PeerJ reviewing PDF | (2021:02:58021:1:0:NEW 12 Apr 2021) 
368

369

370

371

372

373

374

375

376

377

378

379

380

381

382

383

384

385

386

387

388

389

390

391

392

393

394

395

396

397

398

399

400

401

402

403

404

405

406

407

408

409

410

411

412

413

38:367-378.

Gao D, Jimenez-Lopez JC, Iwata A, Gill N, Jackson SA. 2012. Functional and structural divergence of an unusual LTR retrotransposon family in plants. PloS one 7:e48595. DOI: 10.1371/journal.pone.0048595.

Grandbastien M-A. 2015a. LTR retrotransposons, handy hitchhikers of plant regulation and stress response. Biochimica et biophysica acta 1849:403-16. DOI: 10.1016/j.bbagrm.2014.07.017.

Grandbastien M-AA. 2015b. LTR retrotransposons, handy hitchhikers of plant regulation and stress response. Biochimica et Biophysica Acta - Gene Regulatory Mechanisms 1849:403416. DOI: 10.1016/j.bbagrm.2014.07.017.

Hastie T, Tibshirani R, Friedman J. 2009. The elements of statistical learning: data mining, inference, and prediction. Springer Science \& Business Media.

Hesam TD, Ali M-N. 2010. Mining biological repetitive sequences using support vector machines and fuzzy SVM. Iranian Journal of Chemistry and Chemical Engineering 29:117.

Jaiswal AK, Krishnamachari A. 2019. Physicochemical property based computational scheme for classifying DNA sequence elements of Saccharomyces cerevisiae. Computational biology and chemistry 79:193-201. DOI: 10.1016/j.compbiolchem.2018.12.014.

Jiang S-Y, Ramachandran S. 2013. Genome-wide survey and comparative analysis of LTR retrotransposons and their captured genes in rice and sorghum. PloS one 8:e71118. DOI: 10.1371/journal.pone.0071118.

Jurka J, Kapitonov V V., Pavlicek A, Klonowski P, Kohany O, Walichiewicz J. 2005. Repbase Update, a database of eukaryotic repetitive elements. Cytogenetic and Genome Research 110:462-467. DOI: 10.1159/000084979.

Kauer G, Blöcker H. 2003. Applying signal theory to the analysis of biomolecules. Bioinformatics 19:2016-2021.

Komer B, Bergstra J, Eliasmith C. 2014. Hyperopt-Sklearn: Automatic Hyperparameter Configuration for Scikit-Learn. Scipy 2014:33-39.

Larrañaga P, Calvo B, Santana R, Bielza C, Galdiano J, Inza I, Lozano JA, Armañanzas R, Santafé G, Pérez A, Robles V. 2006. Machine learning in bioinformatics. Briefings in Bioinformatics 7:86-112. DOI: 10.1093/bib/bbk007.

Li H, Tian S, Li Y, Fang Q, Tan R, Pan Y, Huang C, Xu Y, Gao X. 2020. Modern deep learning in bioinformatics. Journal of molecular cell biology.

Libbrecht MW, Noble WS. 2015. Machine learning applications in genetics and genomics. Nature Reviews Genetics 16:321-332. DOI: 10.1038/nrg3920.

Loureiro T, Camacho R, Vieira J, Fonseca NA. 2013a. Improving the performance of Transposable Elements detection tools. Journal of integrative bioinformatics 10:231. DOI: 10.2390/biecoll-jib-2013-231.

Loureiro T, Camacho R, Vieira J, Fonseca NA. 2013b. Improving the performance of Transposable Elements detection tools. Journal of integrative bioinformatics 10:231. DOI: 10.2390/biecoll-jib-2013-231.

Mapleson D, Garcia Accinelli G, Kettleborough G, Wright J, Clavijo BJ. 2017. KAT: a K-mer analysis toolkit to quality control NGS datasets and genome assemblies. Bioinformatics 33:574-576.

McCarthy EM, McDonald JF. 2003. LTR STRUC: A novel search and identification program for LTR retrotransposons. Bioinformatics 19:362-367. DOI: 10.1093/bioinformatics/btf878. 
414

415

416

417

418

419

420

421

422

423

424

425

426

427

428

429

430

431

432

433

434

435

436

437

438

439

440

441

442

443

444

445

446

447

448

449

450

451

452

453

454

455

456

457

458

459
Müller AC, Guido S, others. 2016. Introduction to machine learning with Python: a guide for data scientists. "O'Reilly Media, Inc."

Mustafin RN, Khusnutdinova EK. 2018. The Role of Transposons in Epigenetic Regulation of Ontogenesis. Russian Journal of Developmental Biology 49:61-78. DOI: 10.1134/S1062360418020066.

Nair AS, Sreenadhan SP. 2006. A coding measure scheme employing electron-ion interaction pseudopotential (EIIP). Bioinformation 1:197.

Nakano FK, Mastelini SM, Barbon S, Cerri R. 2018a. Improving Hierarchical Classification of Transposable Elements using Deep Neural Networks. In: Proceedings of the International Joint Conference on Neural Networks. Rio de Janeiro, Brazil,.

Nakano FK, Mastelini SM, Barbon S, Cerri R. 2018b. Improving Hierarchical Classification of Transposable Elements using Deep Neural Networks. In: Proceedings of the International Joint Conference on Neural Networks. Rio de Janeiro, Brazil: IEEE,

Nakano FK, Pinto WJ, Pappa GL, Cerri R. 2017. Top-down strategies for hierarchical classification of transposable elements with neural networks. In: Proceedings of the International Joint Conference on Neural Networks. Anchorage, AK, United states, 25392546.

Neumann P, Novák P, Hoštáková N, MacAs J. 2019. Systematic survey of plant LTRretrotransposons elucidates phylogenetic relationships of their polyprotein domains and provides a reference for element classification. Mobile DNA 10:1. DOI: 10.1186/s13100018-0144-1.

Nicolas J, Peterlongo P, Tempel S. 2016. Finding and Characterizing Repeats in Plant Genomes. In: Edwards D ed. Plant Bioinformatics. Springer, 293-337. DOI: 10.1007/978-1-49393167-5_17.

Nordberg H, Cantor M, Dusheyko S, Hua S, Poliakov A, Shabalov I, Smirnova T, Grigoriev I V., Dubchak I. 2014. The genome portal of the Department of Energy Joint Genome Institute: 2014 updates. Nucleic Acids Research 42:26-31. DOI: 10.1093/nar/gkt1069.

Orozco-Arias S, Isaza G, Guyot R. 2019. Retrotransposons in Plant Genomes: Structure, Identification, and Classification through Bioinformatics and Machine Learning. International Journal of Molecular Sciences 20:1-29. DOI: 10.3390/ijms20153837.

Orozco-Arias S, Isaza G, Guyot R, Tabares-soto R. 2019. A systematic review of the application of machine learning in the detection and classi fi cation of transposable elements. Peerj 7:18311. DOI: 10.7717/peerj.8311.

Orozco-Arias S, Jaimes PA, Candamil MS, Jiménez-Varón CF, Tabares-soto R, Isaza G, Guyot R. 2021. InpactorDB : A Classified Lineage-Level Plant LTR Retrotransposon Reference Library for Free-Alignment Methods Based on Machine Learning. MDPI Genes 12:17. DOI: https://doi.org/10.3390/genes12020190.

Orozco-Arias S, Piña JS, Tabares-soto R, Castillo-ossa LF, Guyot R, Isaza G. 2020. Measuring performance metrics of machine learning algorithms for detecting and classifying transposable elements. Processes 8:1-20. DOI: 10.3390/pr8060638.

Orozco-Arias S, Tabares-Soto R, Ceballos D, Guyot R. 2017. Parallel Programming in Biological Sciences, Taking Advantage of Supercomputing in Genomics. In: Solano A, Ordoñez H eds. Advances in Computing. Zurich: Springer, 627-643. DOI: 10.1007/978981-10-2630-0.

Ou S, Chen J, Jiang N. 2018. Assessing genome assembly quality using the LTR Assembly Index (LAI). Nucleic Acids Research:1-11. DOI: 10.1093/nar/gky730.

PeerJ reviewing PDF | (2021:02:58021:1:0:NEW 12 Apr 2021) 
460

461

462

463

464

465

466

467

468

469

470

471

472

473

474

475

476

477

478

479

480

481

482

483

484

485

486

487

488

489

490

491

492

493

494

495

496

497

498

499

500

501

502

503

504

505

Ou S, Su W, Liao Y, Chougule K, Agda JRA, Hellinga AJ, Lugo CSB, Elliott TA, Ware D, Peterson T, Jiang N, Hirsch CN, Hufford MB. 2019. Benchmarking transposable element annotation methods for creation of a streamlined, comprehensive pipeline. Genome biology 20:275. DOI: 10.1186/s13059-019-1905-y.

Pedregosa F, Varoquaux G, Gramfort A, Michel V, Thirion B, Grisel O, Blondel M, Prettenhofer P, Weiss R, Dubourg V, Vanderplas J, Passos A, Cournapeau D, Brucher M, Perrot M, Duchesnay E. 2011. Scikit-learn: Machine Learning in Python. Journal of Machine Learning Research 12:2825-2830.

Price AL, Jones NC, Pevzner PA. 2005. De novo identification of repeat families in large genomes. Bioinformatics 21:351-358. DOI: 10.1093/bioinformatics/bti1018.

Rahman AYA, Usharraj AO, Misra BB, Thottathil GP, Jayasekaran K, Feng Y, Hou S, Ong SY, $\mathrm{Ng}$ FL, Lee LS, others. 2013. Draft genome sequence of the rubber tree Hevea brasiliensis. BMC genomics 14:75.

Rawal K, Ramaswamy R. 2011. Genome-wide analysis of mobile genetic element insertion sites. Nucleic acids research 39:6864-78. DOI: 10.1093/nar/gkr337.

Rigal M, Mathieu O. 2011. A "mille-feuille" of silencing: Epigenetic control of transposable elements. Biochimica et Biophysica Acta (BBA) - Gene Regulatory Mechanisms 1809:452458. DOI: 10.1016/j.bbagrm.2011.04.001.

Rishishwar L, Wang L, Clayton EA, Mariño-ramírez L, Mcdonald JF, Jordan IK, Rishishwar L, Wang L, Clayton EA, Mariño- L, Mcdonald JF, Population IKJ, Wang L, Clayton EA, Mariño-ramírez L, John F, Jordan IK, Jordan IK. 2017. Population and clinical genetics of human transposable elements in the ( post ) genomic era. DOI: 10.1080/2159256X.2017.1280116.

Rosen GL. 2006. Signal processing for biologically-inspired gradient source localization and DNA sequence analysis. Georgia Institute of Technology.

Schietgat L, Vens C, Cerri R, Fischer CN, Costa E, Ramon J, Carareto CMA, Blockeel H. 2018 a. A machine learning based framework to identify and classify long terminal repeat retrotransposons. PLoS computational biology 14:e1006097. DOI: 10.1371/journal.pcbi.1006097.

Schietgat L, Vens C, Cerri R, Fischer CN, Costa E, Ramon J, Carareto CMA, Blockeel H. 2018b. A machine learning based framework to identify and classify long terminal repeat retrotransposons. PLOS Computational Biology 14:e1006097. DOI: 10.1371/journal.pcbi.1006097.

Shastry KA, Sanjay HA. 2020. Machine Learning for Bioinformatics. In: Statistical Modelling and Machine Learning Principles for Bioinformatics Techniques, Tools, and Applications. Springer, 25-39.

Simpson JT, Wong K, Jackman SD, Schein JE, Jones SJM, Birol I. 2009. ABySS: a parallel assembler for short read sequence data. Genome research 19:1117-1123. DOI: 10.1101/gr.089532.108.

Spannagl M, Bader K, Pfeifer M, Nussbaumer T, Mayer KFX. 2016a. PGSB/MIPS Plant Genome Information Resources and Concepts for the Analysis of Complex Grass Genomes. In: 165-186. DOI: 10.1007/978-1-4939-3167-5_8.

Spannagl M, Nussbaumer T, Bader KC, Martis MM, Seidel M, Kugler KG, Gundlach H, Mayer KFX. 2016b. PGSB plantsDB: Updates to the database framework for comparative plant genome research. Nucleic Acids Research 44:D1141-D1147. DOI: 10.1093/nar/gkv1130.

Sun H, Ding J, Piednoël M, Schneeberger K. 2018. findGSE: estimating genome size variation

PeerJ reviewing PDF | (2021:02:58021:1:0:NEW 12 Apr 2021) 
506

507

508

509

510

511

512

513

514

515

516

517

518

519

520

521

522

523

524

525

526

527

528

529

530

531 within human and Arabidopsis using k-mer frequencies. Bioinformatics 34:550-557.

Tabares-soto R, Orozco-Arias S, Romero-Cano V, Segovia Bucheli V, Rodríguez-Sotelo JL, Jiménez-Varón CF. 2020. A comparative study of machine learning and deep learning algorithms to classify cancer types based on microarray gene expression. Peerj Computer Science 6:1-22. DOI: 10.7717/peerj-cs.270.

Wicker T, Sabot F, Hua-Van A, Bennetzen JL, Capy P, Chalhoub B, Flavell A, Leroy P, Morgante M, Panaud O, Paux E, SanMiguel P, Schulman AH. 2007. A unified classification system for eukaryotic transposable elements. Nature Reviews Genetics 8:973982. DOI: $10.1038 / \mathrm{nrg} 2165$.

Witte C-P, Le QH, Bureau T, Kumar A. 2001. Terminal-repeat retrotransposons in miniature (TRIM) are involved in restructuring plant genomes. Proceedings of the National Academy of Sciences 98:13778-13783.

Wold S, Esbensen K, Geladi P. 1987. Principal component analysis. Chemometrics and intelligent laboratory systems 2:37-52.

Yan H, Bombarely A, Li S. 2020. DeepTE: a computational method for de novo classification of transposons with convolutional neural network. Bioinformatics (Oxford, England).

Yu N, Guo X, Gu F, Pan Y. 2015. DNA AS X: An information-coding-based model to improve the sensitivity in comparative gene analysis. In: International Symposium on Bioinformatics Research and Applications. 366-377.

Zerbino DR, Birney E. 2008. Velvet: algorithms for de novo short read assembly using de Bruijn graphs. Genome research 18:821-829.

Zhang C, Ma Y. 2012. Ensemble machine learning: methods and applications. Boston, MA: Springer. DOI: https://doi.org/10.1007/978-1-4419-9326-7.

Zou J, Huss M, Abid A, Mohammadi P, Torkamani A, Telenti A. 2018. A primer on deep learning in genomics. Nature Genetics. DOI: 10.1038/s41588-018-0295-5. 


\section{Table 1 (on next page)}

Machine Learning models and hyper-parameters tuned. 


\begin{tabular}{|c|c|c|}
\hline Classifier & Parameter & Range \\
\hline \multirow{4}{*}{ KNN } & neighbors & $2,20,39,57,76,94,113,131,150$ \\
\hline & weights & uniform, distance \\
\hline & metric & $\begin{array}{c}\text { euclidean, manhattan, chebyshev, } \\
\text { minkowski,wminkowski, seuclidean, } \\
\text { mahalanobis }\end{array}$ \\
\hline & algorithm & auto, ball_tree, kd_tree, brute \\
\hline \multirow{4}{*}{ Linear SVC } & C & $1 \times 10^{i}$ with $i=-4$, to 5 \\
\hline & penalty & 11,12 \\
\hline & loss & hinge, squared_hinge \\
\hline & tol & $10^{-1}, 10^{-2}, 10^{-4}, 10^{-8}$ \\
\hline \multirow{5}{*}{ LR } & $\mathrm{C}$ & $1 \times 10^{i}$ with $i=-4$, to 5 \\
\hline & tol & $1 \times 10^{i}$ with $i=-4$, to 5 \\
\hline & max_iter & $1 \times 10^{i}$ with $i=0$, to 6 \\
\hline & penalty & 11,12 , elasticet, none \\
\hline & solver & $\begin{array}{c}\text { saga, liblinear, newton-cg, lbfgs, sag, } \\
\text { saga }\end{array}$ \\
\hline \multirow{3}{*}{ LDA } & shrinkage & $1,0.1,0.5,0.001,0.0001,0.00001$ \\
\hline & solver & svd, Isqr, eigen \\
\hline & tol & $10^{-1}, 10^{-2}, 10^{-4}, 10^{-8}$ \\
\hline
\end{tabular}

1

2 
Table 2 (on next page)

Composition of the negative instances dataset 


\begin{tabular}{|c|c|c|c|}
\hline TE type & Order & Superfamily & Number \\
\hline \multirow{8}{*}{$\begin{array}{c}\text { Class I } \\
\text { (retrotransposons) }\end{array}$} & Non-LTR & LINES & 767 \\
\hline & Non-LTR & SINES & 551 \\
\hline & PLEs & Penelope & 297 \\
\hline & DIRS & DIRS & 356 \\
\hline & DIRS & VIPER & 1 \\
\hline & DIRS & Ngaro & 2 \\
\hline & Unclassified & Unclassified & 1039 \\
\hline & \multicolumn{2}{|c|}{ TOTAL } & 3013 \\
\hline \multirow{14}{*}{$\begin{array}{l}\text { Class II (DNA } \\
\text { transposons) }\end{array}$} & TIR & Tc1-Mariner & 2326 \\
\hline & TIR & hAT & 2587 \\
\hline & TIR & Merlin & 65 \\
\hline & TIR & Transib & 119 \\
\hline & TIR & PiggyBac & 19 \\
\hline & TIR & PIF - Harbinger & 973 \\
\hline & TIR & MuDR & 1016 \\
\hline & TIR & CACTA & 57 \\
\hline & TIR & En-Spm & 1265 \\
\hline & TIR & MITE & 2312 \\
\hline & Crypton & Crypton & 231 \\
\hline & Helitron & Helitron & 740 \\
\hline & Unclassified & Unclassified & 22,354 \\
\hline & \multicolumn{2}{|c|}{ TOTAL } & 34,064 \\
\hline \multirow{4}{*}{ Non-TEs } & \multicolumn{2}{|c|}{ RNA } & 262,925 \\
\hline & \multicolumn{2}{|c|}{ CDS } & $2,713,028$ \\
\hline & \multicolumn{2}{|c|}{ Quimeric sequences } & 3566 \\
\hline & \multicolumn{2}{|c|}{ TOTAL } & $2,979,519$ \\
\hline
\end{tabular}


Table 3 (on next page)

Tuned parameter values. 


\begin{tabular}{|c|c|c|}
\hline Classifier & Parameter & Value \\
\hline \multirow{4}{*}{ KNN } & neighbors & 2 \\
\cline { 2 - 3 } & weights & distance \\
\cline { 2 - 3 } & metric & euclidean \\
\cline { 2 - 3 } & algorithm & auto \\
\hline \multirow{4}{*}{ Linear SVC } & C & 0.001 \\
\cline { 2 - 3 } & penalty & I2 \\
\cline { 2 - 3 } & loss & squared_hinge \\
\cline { 2 - 3 } & tol & 0.1 \\
\hline \multirow{4}{*}{ LR } & C & 0.01 \\
\cline { 2 - 3 } & tol & 10 \\
\cline { 2 - 3 } & max_iter & 1000 \\
\cline { 2 - 3 } & penalty & I2 \\
\cline { 2 - 3 } & solver & sag \\
\hline \multirow{4}{*}{ LDA } & shrinkage & 0.0001 \\
\cline { 2 - 3 } & solver & Isqr \\
\cline { 2 - 3 } & tol & 0.1 \\
\hline
\end{tabular}

1 
Table 4 (on next page)

Dataset composition used in the binary plus multiclass classification task. 


\begin{tabular}{|c|c|c|c|}
\hline Class & Superfamily & Name & Number of classes \\
\hline 0 & - & $\begin{array}{c}\text { Other genomic } \\
\text { features }\end{array}$ & 34,823 \\
\hline 1 & Copia & ALE/Retrofit & 12,031 \\
\hline 3 & Copia & Angela & 1,458 \\
\hline 4 & Copia & Bianca & 1,827 \\
\hline 8 & Copia & Ikeros & 84 \\
\hline 9 & Copia & Ivana/Oryco & 3,556 \\
\hline 12 & Copia & Tork/Tar & 6,180 \\
\hline 13 & Copia & SIRE & 3,130 \\
\hline & & Total Copia & $\mathbf{2 8 , 2 6 6}$ \\
\hline 14 & Gypsy & CRM & 2,136 \\
\hline 16 & Gypsy & Galadriel & 549 \\
\hline 17 & Gypsy & Reina & 4,532 \\
\hline 18 & Gypsy & Tekay/DEL & 10,396 \\
\hline 19 & Gypsy & Athila & 3,499 \\
\hline 20 & Gypsy & TAT & 17,927 \\
\hline & & Total Gypsy & $\mathbf{3 9 , 0 3 9}$ \\
\hline
\end{tabular}




\section{Figure 1}

Schematic representation of the different approaches used in this study in the binary and multiclass classification tasks in LTR retrotransposons through Machine Learning.

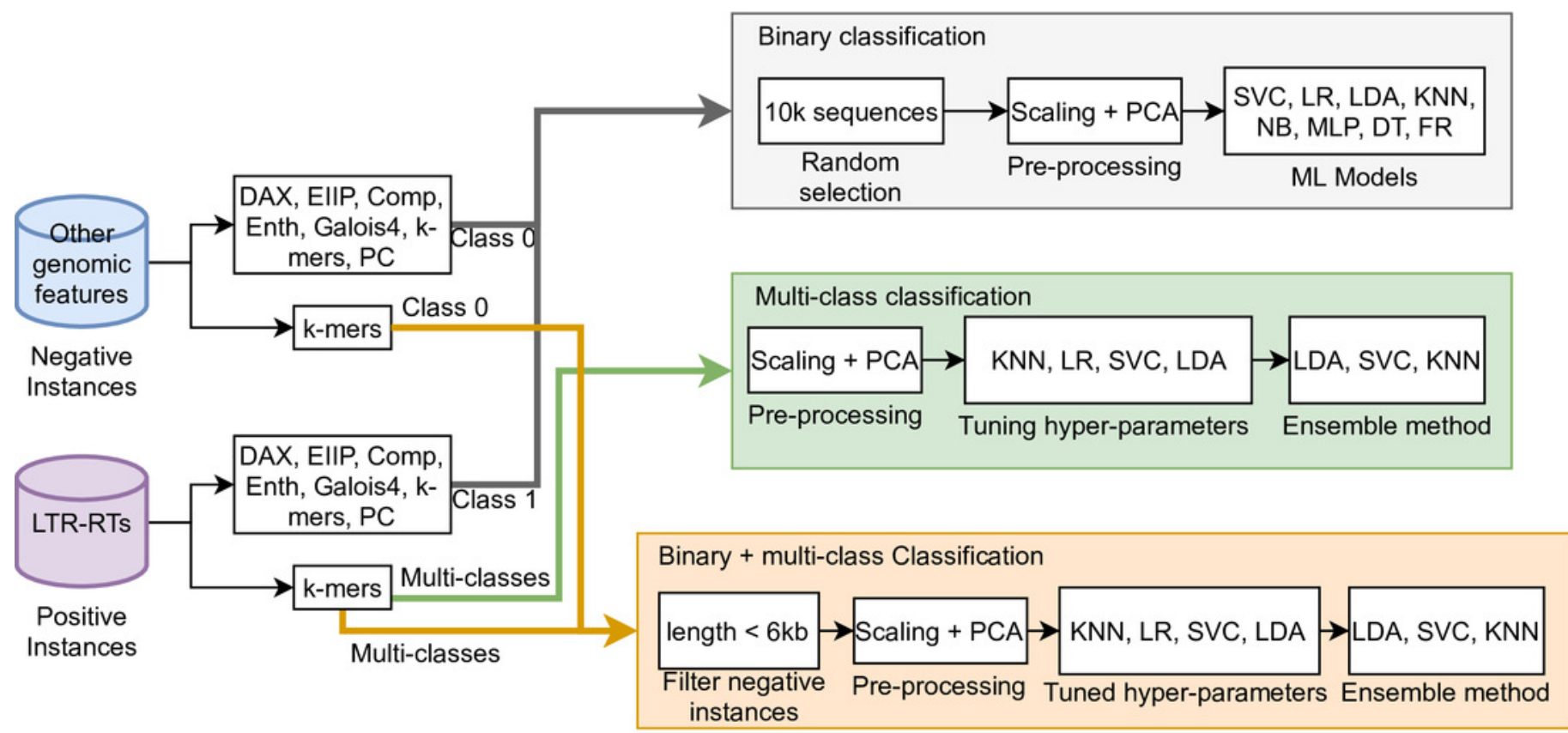


Figure 2

F1-Scores obtained by Machine Learning models using different coding schemes for the test set.

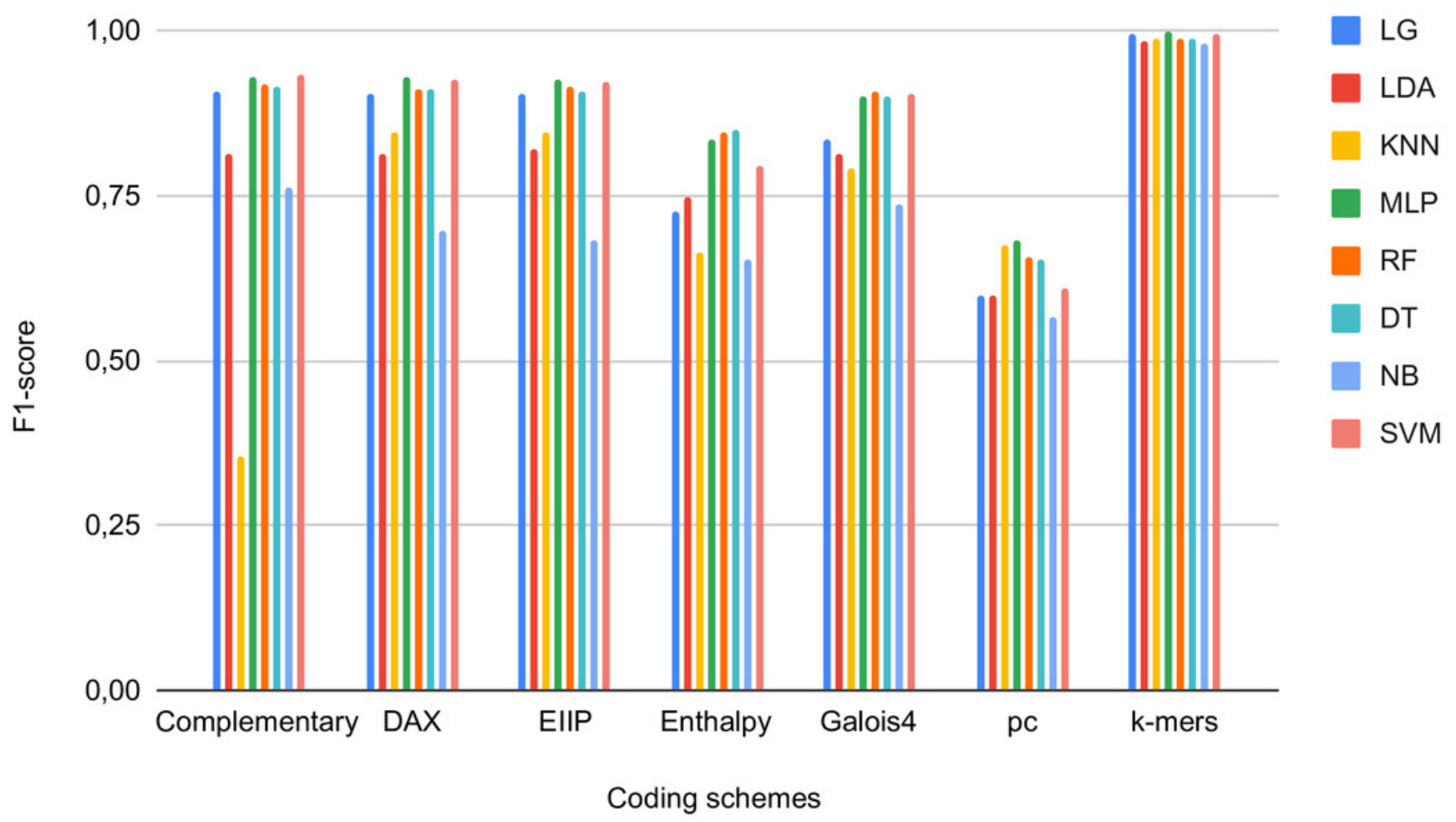


Figure 3

Learning curves of tuned ML algorithms: LR (A), KNN (B), LDA (C), and Linear SVC (D), to classify LTR retrotransposons up to the lineage level. The shadow on the lines indicates the standard variation
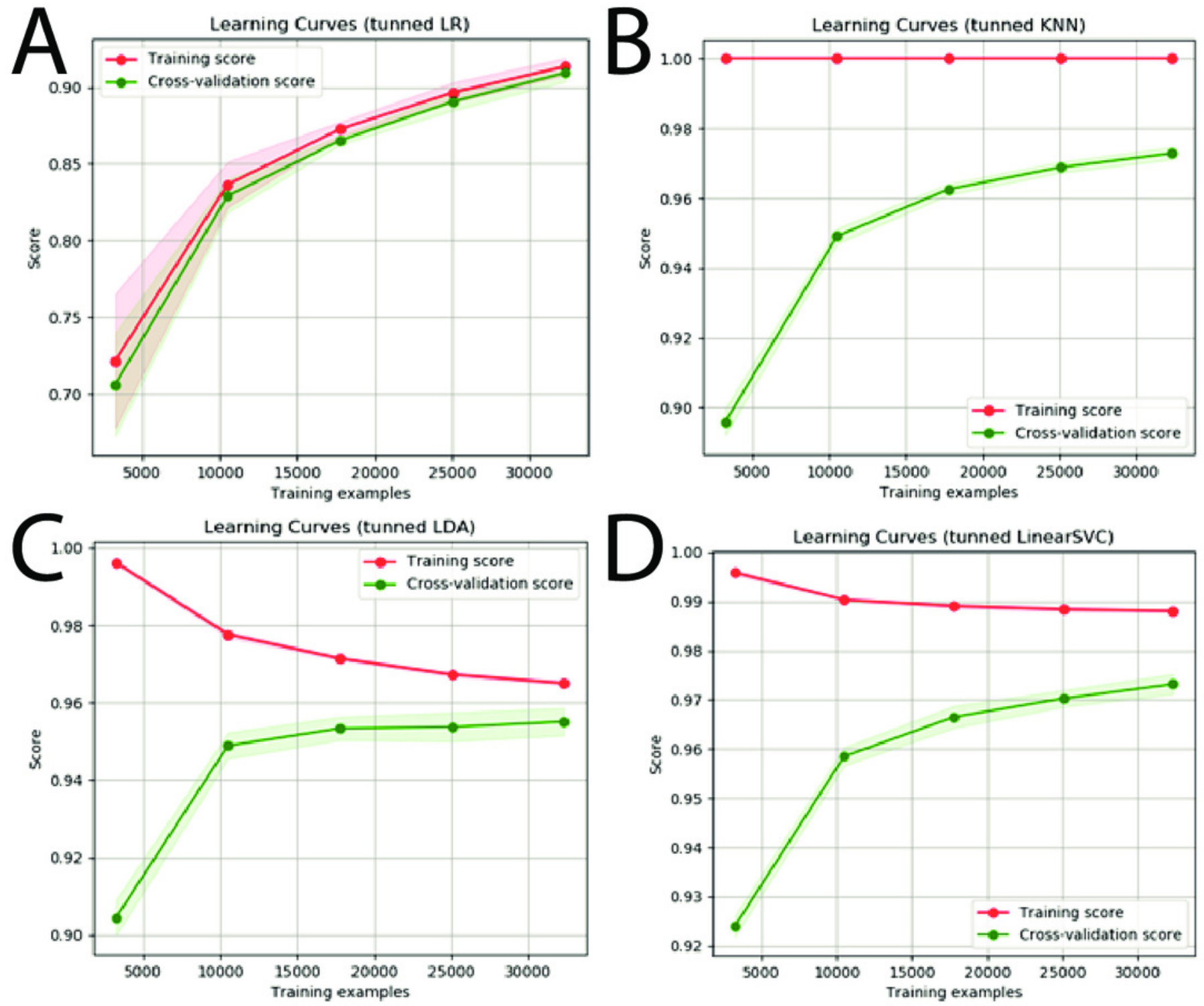
Figure 4

Learning curves of the Stacking classifier algorithm in the classification of LTR retrotransposons up to lineage/family level. The shadow on the line indicates the standard variation.

Learning Curves (tunned Stacking)

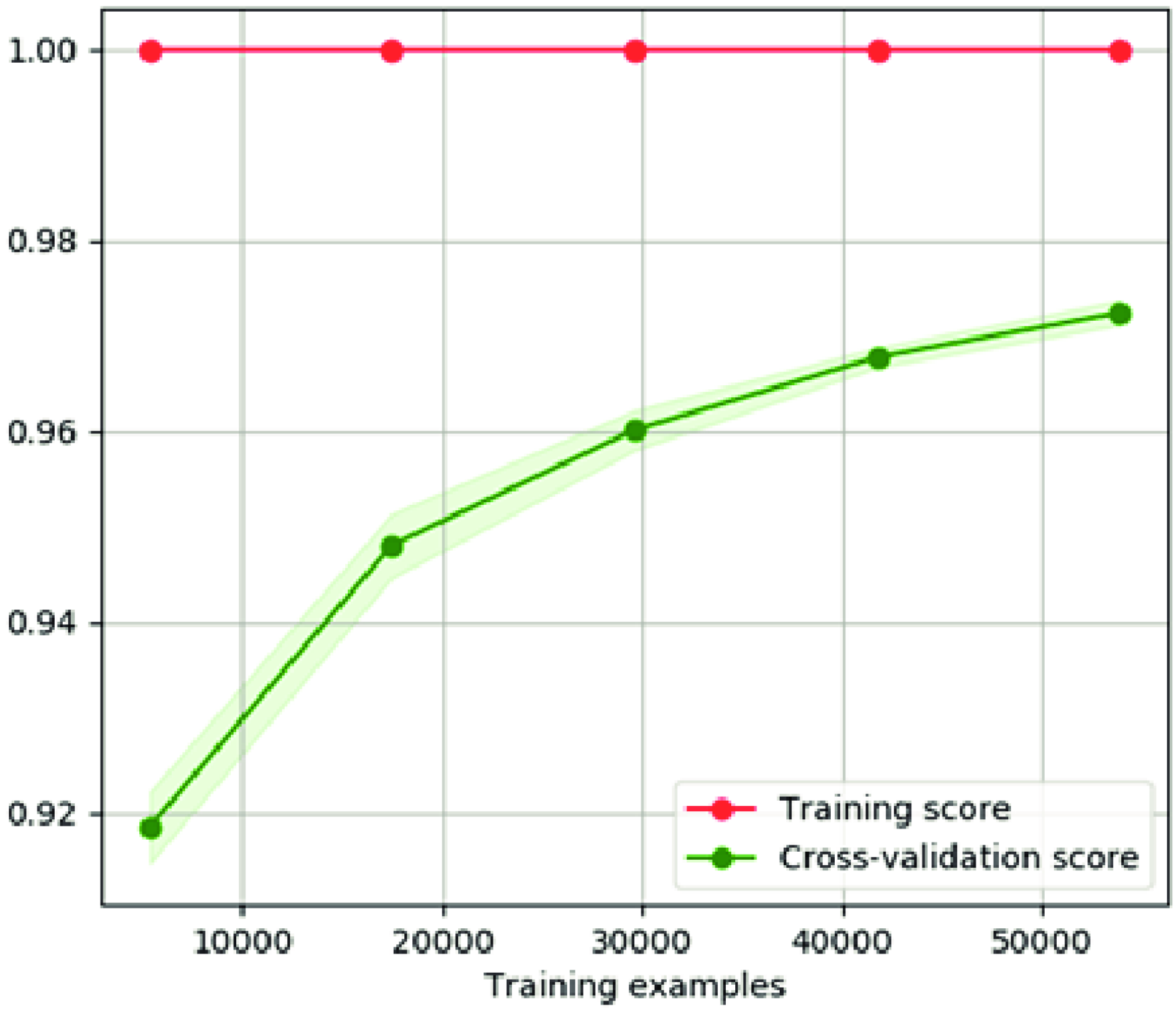


Figure 5

Learning curves for KNN (A), LDA (B), and LR (C) for the binary plus multiclass classification task. The shadow on the lines indicates the standard variation.
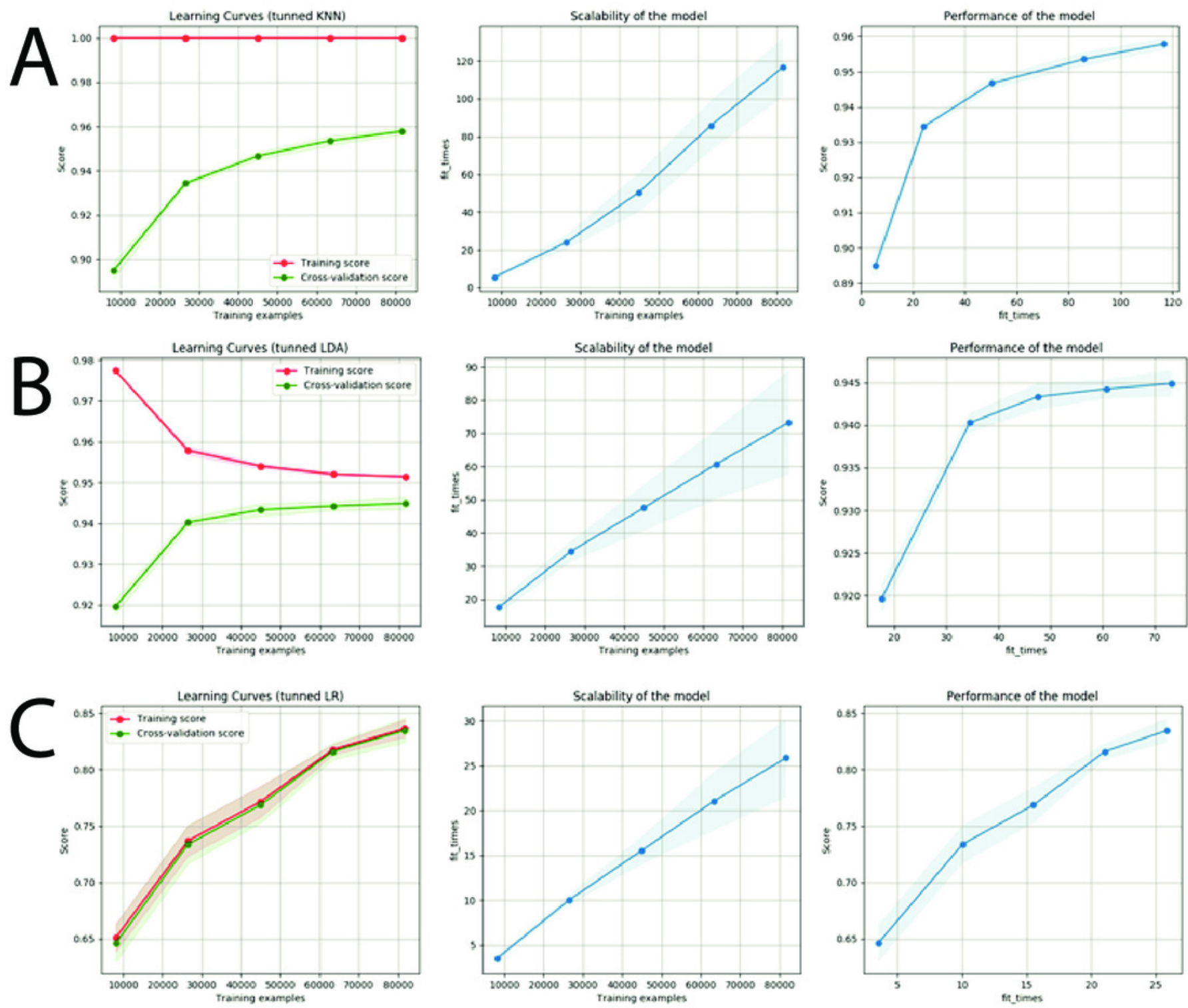
Figure 6

Learning curves for the Staking Classifier (ensemble method) for the binary plus multiclass classification task. The shadow on the lines indicates the standard variation.
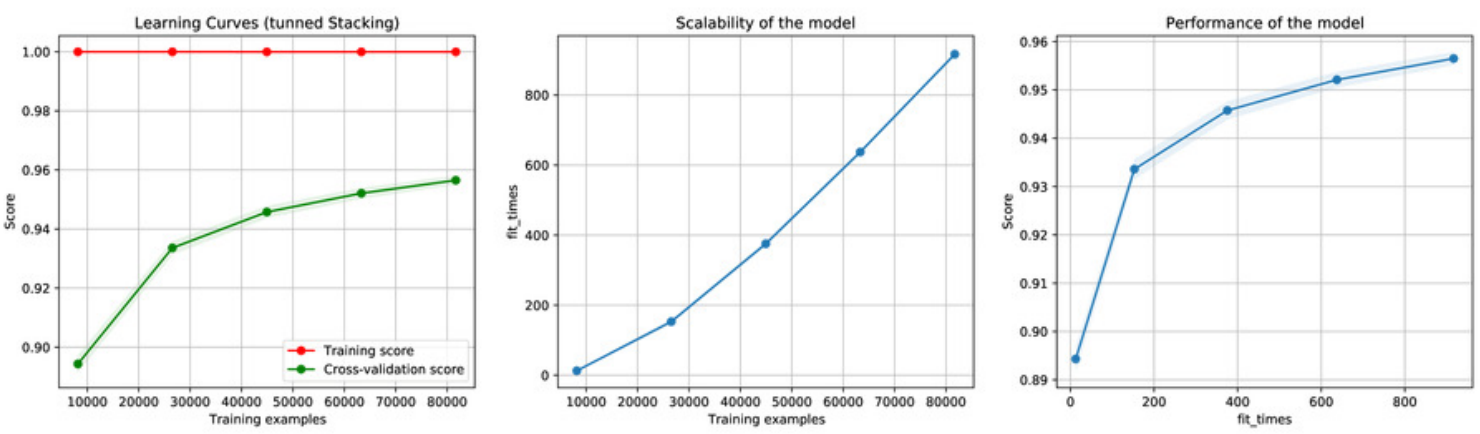
Figure 7

Confusion matrix for the Staking Classifier (ensemble method) used for the binary plus multiclass classification task.

Each class (between 0 and 20) corresponds to the negative instances (for class 0 ) or to a linage/family otherwise (See table 4).

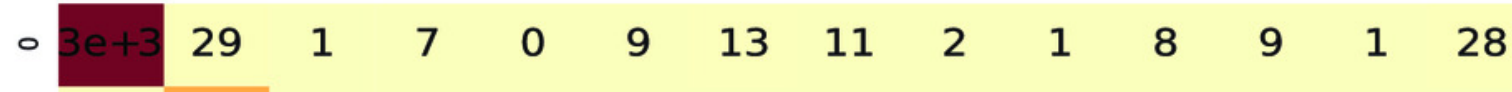

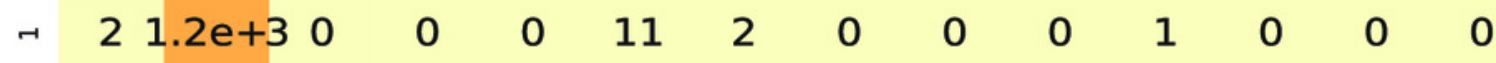

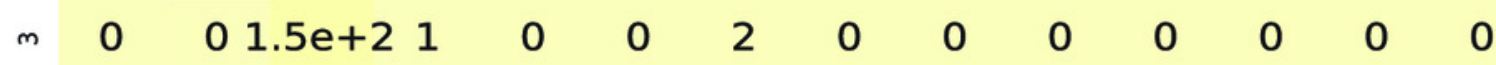

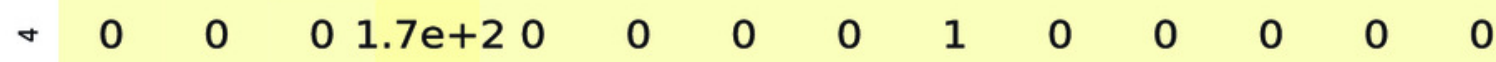

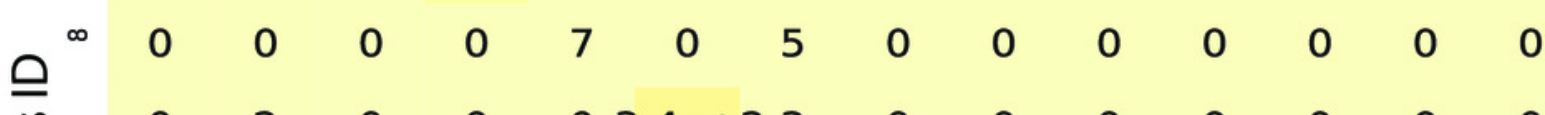

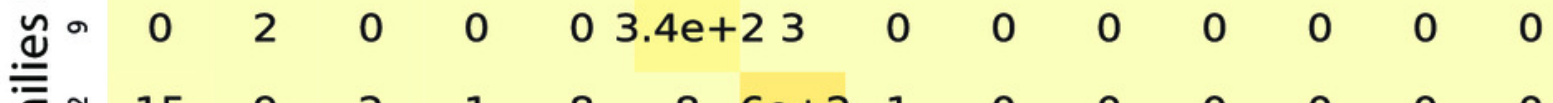

$\begin{array}{lllllllllllllll} & 15 & 9 & 2 & 1 & 8 & 8 & 6 \mathrm{e}+2 & 1 & 0 & 0 & 0 & 0 & 0 & 0\end{array}$

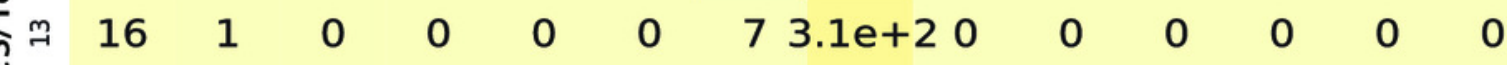

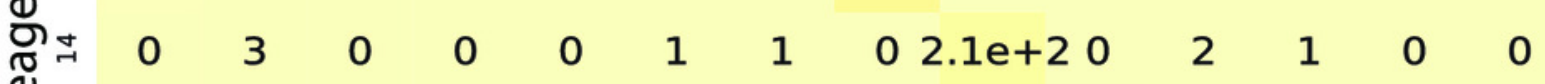

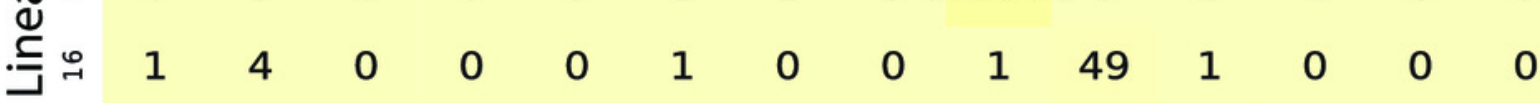

$\begin{array}{llllllllllllll}8 & 22 & 0 & 0 & 0 & 5 & 4 & 0 & 0 & 0 & 4.2 \mathrm{e}+2 & 0 & 0 & 1\end{array}$

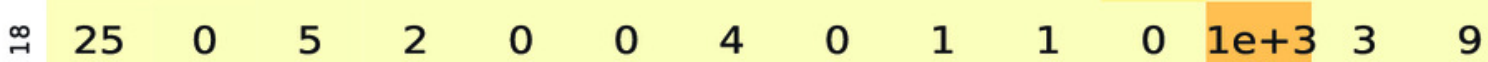

$\begin{array}{lllllllllllllll}2 & 3 & 0 & 0 & 0 & 0 & 0 & 2 & 1 & 3 & 0 & 2 & 0 & 3.2 \mathrm{e}+2 & 0\end{array}$

$\begin{array}{lllllllllllllll} & 59 & 0 & 1 & 0 & 0 & 0 & 0 & 1 & 5 & 0 & 0 & 3 & 3 & 1.8 \mathrm{e}+3\end{array}$

$\begin{array}{lllllllllllllll}0 & 1 & 3 & 4 & 8 & 9 & 12 & 13 & 14 & 16 & 17 & 18 & 19 & 20\end{array}$

Lineages/families ID 
Figure 8

Importance score of the features ( $k$-mer frequencies with $1<=k<=6$ ).

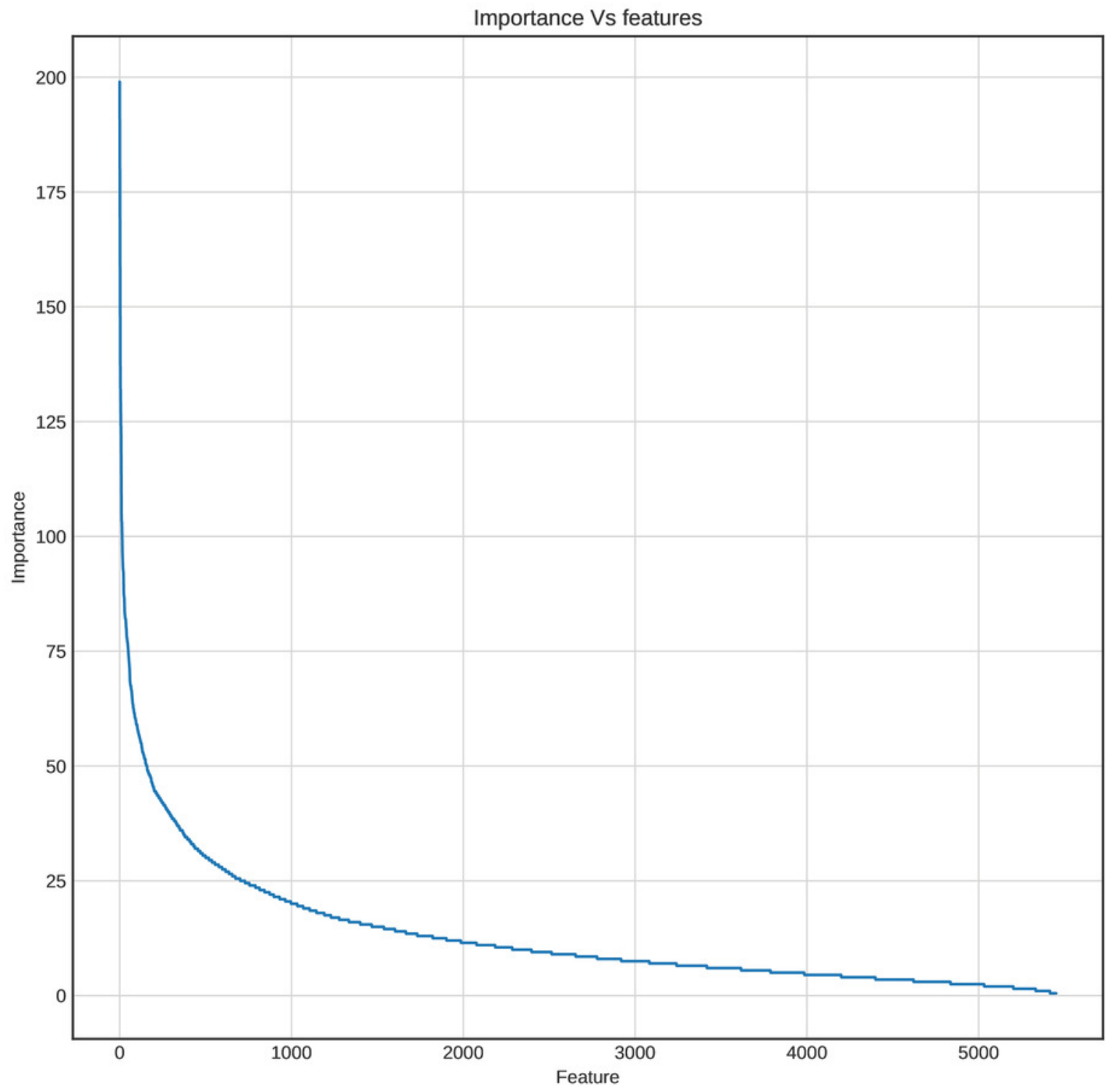


Figure 9

Percentage of features selected based on each value of $k$ (between 1 and 6).

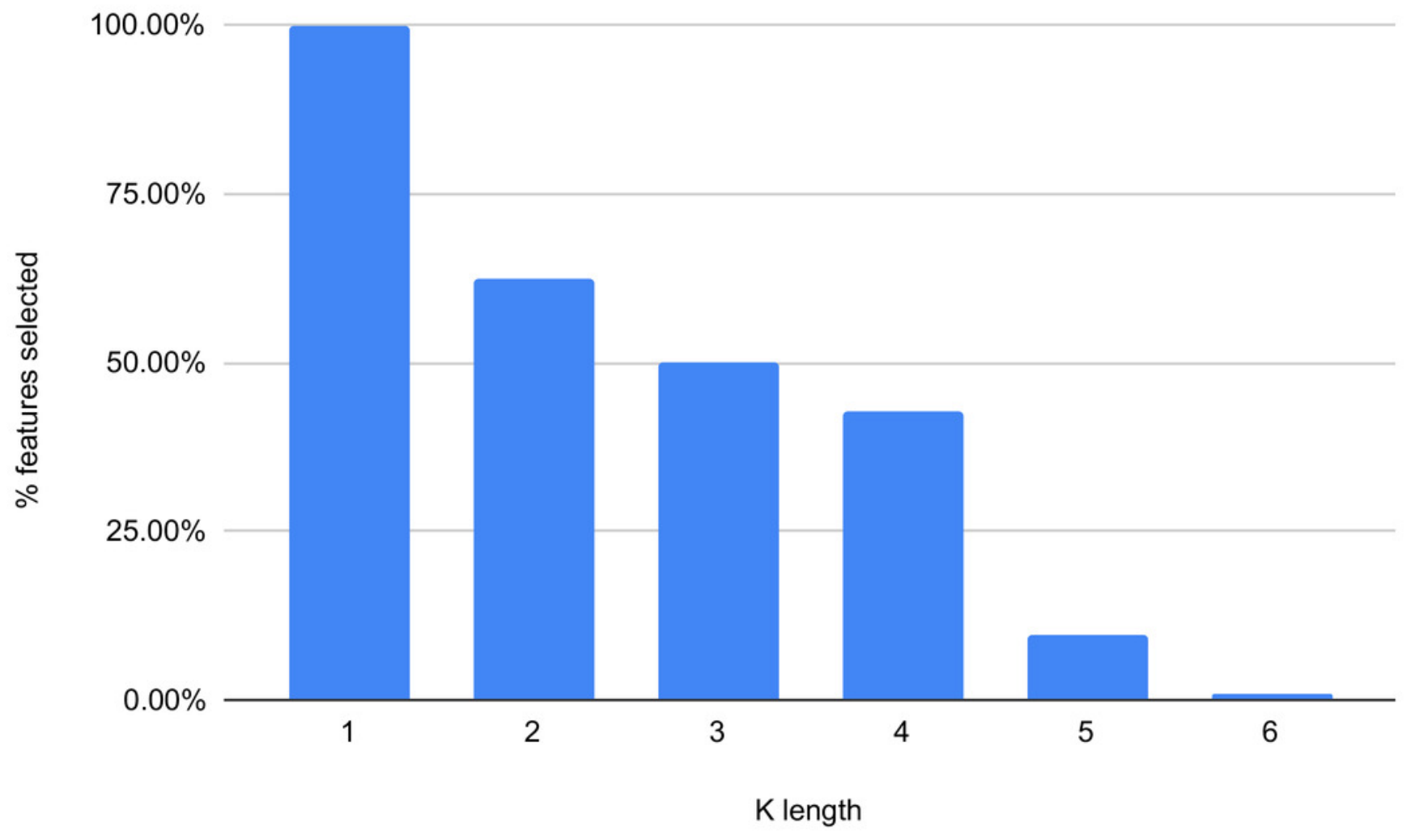


Figure 10

Learning curves for the Staking Classifier (ensemble method) for the binary plus multiclass classification task using only 289 selected features.

The shadow in the lines indicates the standard variation.
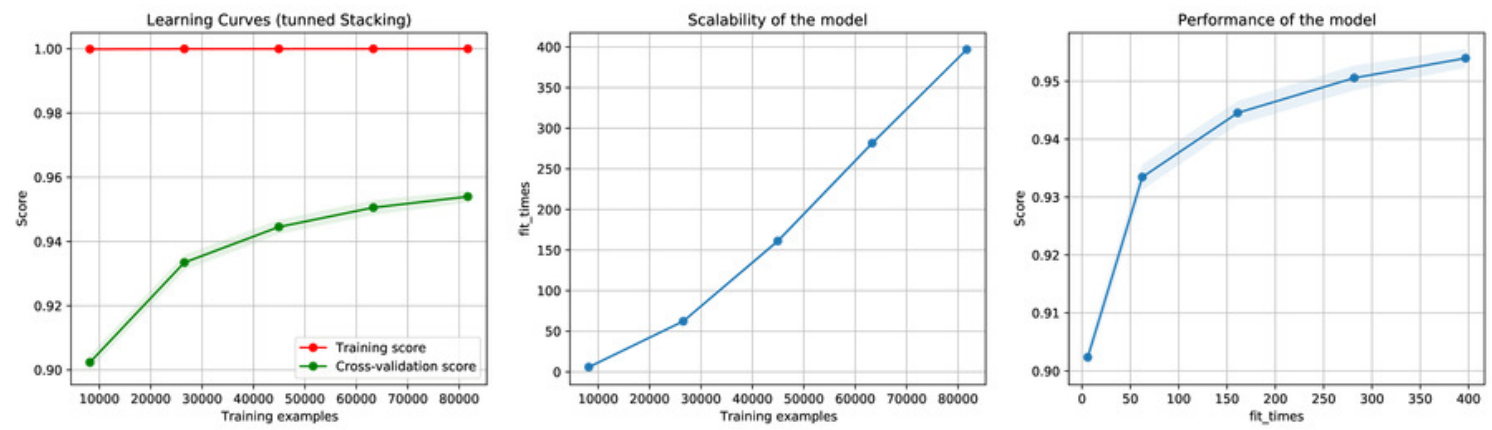